University of Nebraska - Lincoln

DigitalCommons@University of Nebraska - Lincoln

April 1979

\title{
Atomic hydrogen in a uniform magnetic field: Low-lying energy levels for fields below $10^{9} \mathrm{G}$
}

Anthony F. Starace

University of Nebraska-Lincoln, astarace1@unl.edu

Gary L. Webster

University of Nebraska - Lincoln

Follow this and additional works at: https://digitalcommons.unl.edu/physicsstarace

Part of the Physics Commons

Starace, Anthony F. and Webster, Gary L., "Atomic hydrogen in a uniform magnetic field: Low-lying energy levels for fields below $10^{9} \mathrm{G"}$ (1979). Anthony F. Starace Publications. 10.

https://digitalcommons.unl.edu/physicsstarace/10

This Article is brought to you for free and open access by the Research Papers in Physics and Astronomy at DigitalCommons@University of Nebraska - Lincoln. It has been accepted for inclusion in Anthony F. Starace Publications by an authorized administrator of DigitalCommons@University of Nebraska - Lincoln. 


\title{
Atomic hydrogen in a uniform magnetic field: Low-lying energy levels for fields below $10^{9} \mathrm{G}$
}

\author{
Anthony F. Starace and Gary L. Webster \\ Behlen Laboratory of Physics, The University of Nebraska, Lincoln, Nebraska 68588
}

(Received 31 July 1978)

\begin{abstract}
The wave function for an electron in combined Coulomb and uniform magnetic fields is expanded in oblatespheroidal angle functions. The resulting. Schrödinger equation for the radial function is solved for the energy levels by means of two different adiabatic approximations, which yield rigorous lower and upper bounds on the lowest exact energy levels for each symmetry state of the system. Results are presented for the level and binding energies of hydrogenic $1 s, 2 s$, and $2 p$ levels for magnetic fields in the range $10^{7} \leq B \leq 10^{11} \mathrm{G}$ and compared with results of other authors. These results indicate that the present adiabatic approximation methods employing spherical symmetry may be expected to give reliable results for energies of low-lying hydrogenic levels for magnetic fields in the range $0 \leq B \leq 10^{9} \mathrm{G}$.
\end{abstract}

\section{INTRODUCTION}

The theoretical description of a hydrogen atom in a high magnetic field is made difficult by the nonseparability of the Schrödinger equation for an electron in combined Coulomb and uniform magnetic fields. Three general theoretical approaches have been followed: Variational methods have been used by numerous authors ${ }^{1-14}$ to obtain ground- and low-lying excited-state energies and wave functions; eigenfunction-expansion methods $^{15-21}$ have been used, again for the lowest levels, either to diagonalize the Hamiltonian within a finite basis set or to treat a part of the Hamiltonian perturbatively; and adiabatic-approximation methods ${ }^{4,15,22-25}$ have been used, especially for the description of exciton spectra in solids, to separate motion along the magnetic field from that perpendicular to the field. All of these methods are restricted in their applicability: Variational methods, of course, are most useful for calculating upper bounds on the energy of the lowest state of a given symmetry ${ }^{26}$; eigenfunction-expansion methods often require the handling of very large numbers of basis states; and the adiabatic approaches used thus far all employ cylindrical coordinates, thus applying only to very high field strengths (e.g., for atoms, $B \gtrsim 10^{9} \mathrm{G}$ ) and giving a poor description of the wave function in the neighborhood of the origin, where optical absorption takes place.

This paper presents calculations of the $n=1$ and $n=2$ energy levels and binding energies of atomic hydrogen in a uniform magnetic field with strength in the range $10^{7} \leqslant B \lesssim 10^{11} \mathrm{G}$ using an adiabatic approximation in spherical coordinates suggested by Fano. ${ }^{27}$ For the $1 s$ and $2 p$ energy levels our results are rigorous lower bounds. We are also able to compute rigorous upper bounds for these states, but have done so only for the 1 s level, since there are already numerous upper bounds for these states calculated by variational methods. In addition, we have calculated energies for the $2 s$ level of atomic hydrogen. All our calculated energy levels are obtained by solving a one-dimensional differential equation in the radial coordinate $r$. For fields $B \lesssim 10^{9} \mathrm{G}$ our computed energies agree with those of other calculations. A preliminary report on the present results has been given elsewhere. ${ }^{28}$

The main purpose of the energy-level calculations presented here is to test the suitability of an adiabatic approach in spherical coordinates for the description of an atom in a uniform magnetic field. For a quantum-mechanical system, of course, the energy-level spectrum is determined by the asymptotic behavior of the system's wave function. For low-lying levels of hydrogen, our calculations seem to indicate that, even for fields $B \lesssim 10^{9} \mathrm{G}$, a spherical-coordinate description is appropriate. (This may be understood by noting that at $10^{9} \mathrm{G}$ the magnetic energy $e \hbar B / m c$ is 0.43 a.u., which is almost equal to the binding energy of the $1 s$ level of hydrogen, indicating that below $10^{9} \mathrm{G}$ the Coulomb attraction of the nucleus is dominant, at least for the $1 \mathrm{~s}$ level.) Furthermore, the use of spherical coordinates should ensure a good description of the electron's wave function near the origin, where optical absorption takes place. Thus we should expect that the adiabatic wave functions presented here will allow accurate computation of hydrogenic oscillator strengths between low-lying levels in the presence of a uniform magnetic field, which is a topic of some importance in astrophysics. ${ }^{19}$ The key simplification of the adiabatic approach presented here is that the angular part of the electron's wave function is represented by a single oblate-spheroidal angle

1629 (c) 1979 The American Physical Society 
function..$^{29,30}$ This angle function depends parametrically on the radial coordinate $r$ in such a way that at large distances it represents implicitly a linear combination of a large number of spherical harmonics. Thus the oblate-spheroidal angle function implicitly takes into account much of the interaction between states of differing orbital angular momenta (i.e., $l$ mixing) that occurs owing to the quadratic Zeeman part of the Hamiltonian.

Some further developments of the theoretical approach presented in this paper are in progress whose aim is to describe both the high Rydberg and continuum spectra of hydrogenlike atoms in uniform magnetic fields. The principal motivation for these further developments is the possibility for comparison with recent experimental data ${ }^{31-35}$ that have been obtained in these energy regions for magnetic fields of the order of $10^{5} \mathrm{G}$. One such development ${ }^{36}$ of the theory aims to describe the spectral region of moderately high principal quantum numbers $(20 \leqslant n \leqslant 30)$ by allowing for the coupling of a number of adiabatic channels, thereby requiring the solution of a number of coupled differential equations. Another development ${ }^{37}$ of the theory aims to describe the "quasi-Landau" spectral region ${ }^{31,38,39}$ in the vicinity of the zerofield ionization threshold by superposing a very large number of adiabatic states to form the harmonic-oscillator-like state of the electron characteristic of this spectral region.

In Sec. II we present an exact theoretical formulation in spherical coordinates for a hydrogen atom in a uniform magnetic field using oblatespheroidal angle functions and their eigenvalues. Alternative adiabatic approximations are discussed in Sec. III. Properties of the oblate-spheroidal angle functions and eigenvalues necessary for our calculations are reviewed in Sec. IV. Our results for low-lying energy levels of atomic hydrogen and their binding energies in uniform fields in the range $0 \leqslant B \leqslant 10^{11} \mathrm{G}$ are presented in Sec. $\mathrm{V}$ and compared with the results of others. An Appendix proves upper- and lower-bound theorems for some of the energies computed by the adiabatic approaches presented in Sec. III.

Owing to the existence of a number of reviews of the properties of atoms and solids in magnetic fields, this paper does not discuss alternative calculational methods or practical applications of the theory. The most comprehensive and recent review is that by Garstang, ${ }^{40}$ who discusses applications to astrophysics extensively. Related work on exciton spectra in solid-state physics has been reviewed by Hasegawa. ${ }^{41}$ A very succinct but clear survey of alternative theoretical approaches has been given by Edmonds. ${ }^{38}$ Lastly, the geometry of the problem of an electron in combined
Coulomb and uniform magnetic fields has been illustrated graphically by Fano ${ }^{27}$ and by Gajewski. ${ }^{42}$

\section{EXACT FORMULATION}

A. Schrödinger equation

The spin-independent Schrödinger equation in spherical coordinates for an electron in combined Coulomb and uniform magnetic fields is ${ }^{43}$

$$
\begin{array}{r}
\left(\frac{-\hbar^{2}}{2 m_{l}} \vec{\nabla}^{2}+\frac{|e|}{2 m_{l} c} B L_{z}+\frac{e^{2}}{8 m_{l} c^{2}} B^{2} r^{2} \sin ^{2} \theta-\frac{e^{2}}{r}\right) \psi(\overrightarrow{\mathrm{r}}) \\
=E \psi(\overrightarrow{\mathrm{r}}), \quad(1)
\end{array}
$$

where the magnetic field $B$ has been oriented along the $z$ axis, $L_{z}$ is the $z$ component of the orbital angular momentum operator, and $m_{e}$ is the electron mass. ${ }^{44}$ The operators in parentheses are those for the kinetic energy, the linear Zeeman shift, the quadratic Zeeman shift, and the Coulomb energy. We shall use atomic units henceforth (i.e., $m_{e}=e=\hbar=1$ ) and introduce the strength parameter $\alpha$ :

$$
\alpha \equiv B / 2 c=\left(2.12715 \times 10^{-10} \text { a.u. } / \mathrm{G}\right) B(\mathrm{G}) .
$$

Equation (1) in atomic units thus becomes

$$
\left(-\frac{1}{2} \vec{\nabla}^{2}+\alpha L_{z}+\frac{1}{2} \alpha^{2} \gamma^{2} \sin ^{2} \theta-1 / r\right) \psi(\overrightarrow{\mathrm{r}})=E \psi(\overrightarrow{\mathrm{r}}) .
$$

Taking account of the azimuthal symmetry of the problem, we substitute

$$
\psi(\overrightarrow{\mathrm{r}}) \equiv\left[\chi_{m}(r, \theta) / r\right][\exp (i m \varphi) / \sqrt{2 \pi}]
$$

into Eq. (3) to get the following equation for $\chi_{m}(r, \theta)$ :

$$
\left(\frac{d^{2}}{d r^{2}}+\frac{2}{r}+2 E^{\prime}-\frac{\Lambda_{m}\left(\alpha r^{2}, \theta\right)}{r^{2}}\right) \chi_{m}(r, \theta)=0,
$$

where the operator $\Lambda_{m}\left(\alpha r^{2}, \theta\right)$ is defined by

$$
\begin{aligned}
\Lambda_{m}\left(\alpha r^{2}, \theta\right) \equiv & -\frac{1}{\sin \theta} \frac{\partial}{\partial \theta}\left(\sin \theta \frac{\partial}{\partial \theta}\right) \\
& +\frac{m^{2}}{\sin ^{2} \theta}+\alpha^{2} r^{4} \sin ^{2} \theta
\end{aligned}
$$

and the energy $E^{\prime}$ by

$$
E^{\prime} \equiv E-\alpha m \text {. }
$$

\section{B. Expansion in oblate-spheroidal angle functions}

The operator defined in Eq. (6), considered to depend parametrically on the quantity

$$
c \equiv \alpha r^{2},
$$

has the oblate-spheroidal angle functions $g_{m \nu}(c, \theta)$ as eigenstates $:^{29,30}$

$$
\Lambda_{m}(c, \theta) g_{m \nu}(c, \theta)=\left[\lambda_{m \nu}(c)+c^{2}\right] g_{m \nu}(c, \theta),
$$


$g_{m \nu}(c, \theta)$ and their associated eigenvalues $\lambda_{m \nu}(c)$. Note that in the limit that the parameter $c$ vanishes (i.e., due either to $r \rightarrow 0$ or to $B \rightarrow 0$ ), the oblate-spheroidal angle functions and eigenvalues reduce to the familiar limits

$$
\begin{aligned}
g_{m \nu}(c, \theta) \underset{c \rightarrow 0}{\rightarrow} \Theta_{\nu m}(\cos \theta) \\
\quad=(-1)^{m}\left(\frac{(2 \nu+1)(\nu-m) !}{2(\nu+m) !}\right)^{1 / 2} P_{\nu}^{m}(\cos \theta),
\end{aligned}
$$

$\lambda_{m \nu}(c) \underset{c \rightarrow 0}{\rightarrow} \nu(\nu+1)$

where $P_{\nu}^{m}(\cos \theta)$ is an associated Legendre polynomial and $\Theta_{\nu m}(\cos \theta)$ is related to the spherical harmonic $Y_{\nu m}(\theta, \phi)$ as follows:

$$
Y_{\nu m}(\theta, \varphi)=\Theta_{\nu m}(\cos \theta)[\exp (i m \varphi)] / \sqrt{2 \pi} .
$$

Note that for finite values of $c$ the index $\nu$ does not specify a particular value for the electron's orbital angular momentum $l$; rather, each $g_{m \nu}$ may be written as a linear sum of $\Theta_{l m}$ functions where the summation is over $l .^{29,30}$ Note also that we employ a notation for the oblate-spheroidal angle functions that is different from the standard one ${ }^{29,30}$ because we employ a different normalization; i.e., we require

$$
\left(g_{m \nu}, g_{m \nu^{\prime}}\right) \equiv \int_{0}^{\pi} g_{m \nu}(c, \theta) g_{m \nu^{\prime}}(c, \theta) \sin \theta d \theta=\delta_{\nu \nu^{\prime}} .
$$

However, we do retain the standard notation $c$ for the parameter on which the angle functions and their eigenvalues depend; note that the speed of light has been eliminated from Eq. (3) and succeeding equations by absorbing it into the strength parameter $\alpha$ defined by Eq. (2). Lastly, we note that $\nu \geqslant|m|$, and that under inversion the parity of the oblate-spheroidal angle function $g_{m \nu}$ is $(-1)^{\nu}$.

The wave function $\chi_{m}(r, \theta)$ [cf. Eqs. (4) and (5)] may be expressed as an expansion in oblate-spheroidal angle functions:

$$
\chi_{m}(r, \theta) \equiv \sum_{\nu^{\prime}}^{\prime} h_{m \nu^{\prime}}(r) g_{m \nu^{\prime}}(c, \theta),
$$

where the prime on the summation symbol indicates that the sum is over either even or odd $\nu^{\prime}$ values (consistent with the condition $\nu^{\prime} \geqslant|m|$ ), since the electron has a well-defined parity in this problem. The normalization of $\psi(\overrightarrow{\mathrm{r}})$ in Eq. (4) to unity and the normalization of the oblatespheroidal angle functions in Eq. (10) imply that the functions $h_{m \nu^{\prime}}(r)$ satisfy the normalization condition

$$
\sum_{\nu^{\prime}} \int_{0}^{\infty} d r h_{m \nu^{\prime}}^{2}(r)=1
$$

Substituting Eq. (11) into Eq. (5), multiplying from the left by $g_{m \nu}(c, \theta)$, and integrating over $\theta$ gives the following set of coupled differential equations for the radial functions $h_{m \nu}(r)$ :

$$
\begin{aligned}
\left(\frac{d^{2}}{d r^{2}}+2 E^{\prime}+\frac{2}{r}\right. & \left.-\frac{\left(\lambda_{m \nu}(c)+c^{2}\right.}{r^{2}}\right) h_{m \nu}(r) \\
& +2 \sum_{\nu^{\prime}}^{\prime}\left(g_{m \nu}, \frac{\partial g_{m \nu^{\prime}}}{\partial r}\right) \frac{d}{d r} h_{m \nu^{\prime}}(r) \\
& +\sum_{\nu^{\prime}}^{\prime}\left(g_{m \nu}, \frac{\partial^{2} g_{m \nu^{\prime}}}{\partial r^{2}}\right) h_{m \nu^{\prime}}(r)=0,
\end{aligned}
$$

where we have used the following notation for the coupling matrix elements:

$$
\begin{aligned}
& \left(g_{m \nu}, \frac{\partial g_{m \nu^{\prime}}}{\partial r}\right) \equiv \int_{0}^{\pi} g_{m \nu}(c, \theta) \frac{\partial}{\partial r} g_{m \nu^{\prime}}(c, \theta) \sin \theta d \theta \\
& \left(g_{m \nu}, \frac{\partial^{2} g_{m \nu^{\prime}}}{\partial r^{2}}\right) \equiv \int_{0}^{\pi} g_{m \nu}(c, \theta) \frac{\partial^{2}}{\partial r^{2}} g_{m \nu^{\prime}}(c, \theta) \sin \theta d \theta .
\end{aligned}
$$

\section{Properties of coupling matrix elements}

Differentiation of the orthonormality equation (10) with respect to $r$ [N.B., in Eq. (10), $c \equiv \alpha r^{2}$ ] shows that the first derivative matrix elements (14a) are antisymmetric:

$$
\left(g_{m \nu}, \frac{\partial g_{m \nu^{\prime}}}{\partial r}\right)=-\left(\frac{\partial g_{m \nu}}{\partial r}, g_{m \nu^{\prime}}\right)=-\left(g_{m \nu^{\prime}}, \frac{\partial g_{m \nu}}{\partial r}\right) \text {. }
$$

This implies that the diagonal matrix elements vanish:

$$
\left(g_{m \nu}, \frac{\partial g_{m \nu}}{\partial r}\right)=0 \text {. }
$$

Similarly, differentiation of the first derivative coupling matrix element [Eq. (14a)] with respect to $r$ results in the following expression for the second derivative coupling matrix element [Eq. (14b)]:

$$
\left(g_{m \nu}, \frac{\partial^{2} g_{m \nu^{\prime}}}{\partial r^{2}}\right)=-\left(\frac{\partial g_{m \nu}}{\partial r}, \frac{\partial g_{m \nu^{\prime}}}{\partial r}\right)+\frac{\partial}{\partial r}\left(g_{m \nu}, \frac{\partial g_{m \nu^{\prime}}}{\partial r}\right) .
$$

The diagonal matrix element is thus seen to be negative definite:

$$
\left(g_{m \nu}, \frac{\partial^{2} g_{m \nu}}{\partial r^{2}}\right)=-\left(\frac{\partial g_{m \nu}}{\partial r}, \frac{\partial g_{m \nu}}{\partial r}\right) \leqslant 0,
$$


owing to Eq. (16) and to the definition

$$
\left(\frac{\partial g_{m \nu}}{\partial r}, \frac{\partial g_{m \nu}}{\partial r}\right) \equiv \int_{0}^{\pi}\left(\frac{\partial g_{m \nu}}{\partial r}\right)^{2} \sin \theta d \theta .
$$

Lastly, note that since the oblate-spheroidal angle functions have a well-defined parity ${ }^{29,30}$ equal to $(-1)^{\nu}$, the coupling matrix elements in Eq. (14) vanish unless $\nu$ and $\nu^{\prime}$ correspond to the same parity. Other properties of and expressions for the matrix elements in Eq. (14) are known, ${ }^{36}$ but are not needed in this paper.

\section{ADIABATIC APPROXIMATIONS}

Solution of the infinite set of coupled differential equations (13) constitutes an exact solution to the problem of an electron in combined Coulomb and uniform magnetic fields. Practically, of course, one obtains an approximate solution by truncating the infinite set of coupled differential equations to some finite set of equations. This approximation amounts to ignoring certain coupling matrix elements of the type found in Eq. (14). In the extreme case, considered here, one ignores all (or nearly all) coupling matrix elements. Ordinarily such a drastic approximation would require justification in the form of a detailed consideration of the magnitude of the coupling matrix elements. In the Appendix, however, we prove that the two adiabatic approximations presented below give rigorous lower and upper bounds to the system's lowest energy level of each symmetry. (The symmetry state of an electron in combined Coulomb and uniform magnetic fields is specified by the $z$ component of the orbital angular momentum, denoted by the quantum number $m$, and by the parity.) Thus the energy levels calculated by the adiabatic approximations below are significant first approximations, even without a detailed analys is of the strength of the coupling matrix elements.

\section{A. Lower-bound adiabatic approximation}

If we neglect all coupling matrix elements in Eq. (13), we obtain

$$
\left(-\frac{1}{2} \frac{d^{2}}{d r^{2}}+\frac{\lambda_{m \nu}(c)+c^{2}}{2 r^{2}}-\frac{1}{r}\right) h_{m \nu}(r)=E^{\prime} h_{m \nu}(r) \text {. }
$$

Solution of Eq. (20) for fixed $m$ and $\nu$ gives a spectrum of eigenvalues $E_{n}^{\prime}$ and eigenfunctions $h_{m \nu n}(r)$. In the Appendix we prove that for a value of $\nu$ appropriate to the lowest energy level for given $m$ and parity (see Table I), the lowest energy $E^{\prime}$ computed from Eq. (20) is a lower bound on the lowest exact energy for the same $m$ parity. Calculation of the exact energy for given $m$ and parity, of
TABLE I. Lowest energy level for fixed $m$ and parity.

\begin{tabular}{rlll}
\hline \hline$m$ & Parity & $\nu^{\mathrm{a}}$ & Designation $^{\mathrm{b}}$ \\
\hline 0 & even & 0 & 1 s $m_{l}=0$ \\
0 & odd & 1 & $2 p m_{l}=0$ \\
\pm 1 & odd & 1 & $2 p m_{l}= \pm 1$ \\
\pm 1 & even & 2 & $3 d m_{l}= \pm 1$ \\
\pm 2 & even & 2 & $3 d m_{l}= \pm 2$ \\
\pm 2 & odd & 3 & $4 f m_{l}= \pm 2$ \\
\pm 3 & odd & 3 & $4 f m_{l}= \pm 3$ \\
\pm 3 & even & 4 & $5 g m_{l}= \pm 3$ \\
\hline \hline
\end{tabular}

${ }^{a}$ For given $m$ and parity, the value of $\nu$ for the lowest energy level is the smallest integer $\nu \geqslant|m|$ such that $(-1)^{\nu}$ is equal to the parity of the state.

${ }^{b}$ The designation is that of the hydrogenic energy level appropriate in the limit of zero magnetic field.

course, requires the solution of the infinite set of coupled differential equations (13). The calculation of the oblate-spheroidal eigenvalue $\lambda_{m \nu}(c)$ appearing in Eq. (20) is discussed in Sec. IV. The hydrogenic designations of the energy levels for which rigorous lower bounds may be obtained are listed in Table I.

\section{B. Upper-bound adiabatic approximation}

If we neglect all off-diagonal coupling matrix elements in Eq. (13), we obtain [cf. Eq. (16)]

$$
\begin{array}{r}
{\left[-\frac{1}{2} \frac{d^{2}}{d r^{2}}+\frac{\lambda_{m \nu}(c)+c^{2}}{2 r^{2}}-\frac{1}{r}-\frac{1}{2}\left(g_{m \nu}, \frac{\partial^{2} g_{m \nu}}{\partial r^{2}}\right)\right] h_{m \nu}(r)} \\
=E^{\prime} h_{m \nu}(r) .(21)
\end{array}
$$

Equation (21) differs from Eq. (20) by the inclusion of the diagonal second derivative coupling matrix element, which from Eq. (18) is seen to contribute a positive-definite upward shift to the energy spectrum. Note that the energies $E^{\prime}$ and radial functions $h_{m \nu}(r)$ in Eq. (21) are different from those in Eq. (20) owing to this additional term. In the Appendix we prove that for a value of $\nu$ appropriate for the lowest energy level for a given $m$ and parity (see Table I), the lowest energy $E^{\prime}$ computed from Eq. (21) is an upper bound on the lowest exact energy for the same $m$ and parity. The calculation of the oblate-spheroidal eigenvalue $\lambda_{m \nu}(c)$ and the diagonal coupling term $\left(g_{m \nu}, \partial^{2} g_{m \nu} /\right.$ $\left.\partial r^{2}\right)$ which appear in Eq. (21) is discussed in Sec. IV. The hydrogenic designations of the energy levels for which rigorous upper bounds may be obtained are listed in Table I.

\section{CALCULATION OF OBLATE-SPHEROIDAL EIGENVALUES AND EIGENFUNCTIONS}

A primary method for calculating the oblatespheroidal eigenfunctions is to expand them in terms of associated Legendre polynomials. ${ }^{29,30}$ 
We have expanded these eigenfunctions in terms of the $\Theta_{l m}(\cos \theta)$ functions, which are related to the associated Legendre polynomials according to Eq. (9a). Thus we write

$$
g_{m \nu}(c, \theta)=\sum_{l^{\prime} \geqslant|m|} D^{m \nu}(c) \Theta_{l^{\prime} m}(\cos \theta),
$$

where, again, $c \equiv \alpha r^{2}$, and the prime on the sum means that only those $l^{\prime}$ values which have the same parity as $\nu$ are summed. In accordance with Eq. (9a), we expect that in the limit of vanishing $c$, the coefficients become

$$
D_{l}^{m \nu}(c) \underset{c \rightarrow 0}{\longrightarrow} \delta_{l \nu}
$$

Since the $\Theta_{l m}(\cos \theta)$ functions are orthonormal in $l$, the normalization for the oblate-spheroidal angle functions $g_{m \nu}$ in Eq. (10) gives the following normalization requirement for the coefficients $D_{l}^{m \nu}(c)$ :

$$
\sum_{l} D_{l}^{m \nu}(c) D_{l}^{m \nu^{\prime}}(c)=\delta_{\nu \nu^{\prime}} \text {. }
$$

Substituting Eq. (22) into Eq. (8), multiplying from the left by $\Theta_{l m}(\cos \theta)$, and integrating over $\theta$, we obtain the three-term recursion relation that is the basis for computing the oblate-spheroidal eigenfunctions and eigenvalues:

$$
\begin{aligned}
{\left[\lambda_{m \nu}(c)+c^{2}\right.} & \left.\left(1-I_{l, l}\right)-l(l+1)\right] D_{l}^{m \nu}(c) \\
& -c^{2} I_{l, l-2} D_{l-2}^{m \nu}(c)-c^{2} I_{l, l+2} D_{l+2}^{m \nu}(c)=0,
\end{aligned}
$$

where we have defined the integral $I_{l, l}$, as follows ${ }^{45}$ :

$$
\begin{aligned}
I_{l, l^{\prime}} & \equiv \int_{0}^{\pi} \Theta_{l m}(\cos \theta) \sin ^{2} \theta \Theta_{l^{\prime} m}(\cos \theta) \sin \theta d \theta \\
& =\delta_{l l^{\prime}} \frac{2}{3}\left(1+\frac{3 m^{2}-l(l+1)}{(2 l+3)(2 l-1)}\right)-\delta_{l^{\prime}, l^{+2}} \frac{1}{2 l+3}\left(\frac{(l+m+2)(l+m+1)(l-m+2)(l-m+1}{(2 l+5)(2 l+1)}\right)^{1 / 2} \\
& -\delta_{l^{\prime}, l-2} \frac{1}{2 l-1}\left(\frac{(l+m)(l+m-1)(l-m)(l-m-1)}{(2 l+1)(2 l-3)}\right)^{1 / 2}
\end{aligned}
$$

Equation (25) is solved by iteration for the eigenvalue $\lambda_{m \nu}(c)$ and the coefficients $D_{l}^{m \nu}(c)$ as described below.

\section{A. Alternative expressions and special cases of Eq. (25)}

While Eq. (24) determines the normalization of the coefficients $D_{l}^{m \nu}(c)$, Eq. (25) determines their relative magnitudes according to the two alternative expressions

$$
\begin{gathered}
\frac{D_{l}^{m \nu}(c)}{D_{l-2}^{m \nu}(c)}=c^{2} I_{l, l-2}\left\{\left[\lambda_{m \nu}(c)+c^{2}\left(1-I_{l, l}\right)-l(l+1)\right]\right. \\
\left.-c^{2} I_{l, l+2} D_{l+2}^{m \nu}(c) / D_{l}^{m \nu}(c)\right\}^{-1}, \\
\frac{D_{l}^{m \nu}(c)}{D_{l+2}(c)}=c^{2} I_{l, l+2}\left\{\left[\lambda_{m \nu}(c)+c^{2}\left(1-I_{l, l}\right)-l(l+1)\right]\right. \\
\left.-c^{2} I_{l, l-2} D_{l-2}^{m \nu}(c) / D_{l}^{m \nu}(c)\right\}^{-1},
\end{gathered}
$$

Each of Eqs. (27) and (28) may be iterated by substituting for the coefficient ratios in the denominators on the right expressions given by Eqs. (27) and (28), respectively. One thus obtains a continued-fraction solution for each coefficient ratio. In Eq. (27) one iterates "upward" far enough so that eventually one may set $D_{l+n+2}^{m \nu}(c) / D_{l+n}^{m \nu}(c)=0$ for some integer $n$ without affecting the calculated value of $D_{i}^{m \nu}(c) / D_{l-2}^{m \nu}(c)$. Generally the integer $n$ increases rapidly with increasing $c$, but the iteration is simple to carry out on a computer. The iteration of Eq. (28) proceeds similarly except that it is "downward" and eventually one arrives at the lower limit of $l$, denoted by $l_{0}$, which is determined by the relations

$$
l_{0} \geqslant|m| \text { and }(-1)^{l_{0}}=(-1)^{\nu} \text {. }
$$

The coefficient $D_{l_{0-2}}^{m \nu}(c)$ vanishes, of course, and Eq. (25) thus gives the ratio of the remaining coefficients as

$$
\begin{aligned}
& D_{l_{0}+2}^{m \nu}(c) / D_{l_{0}}^{m \nu}(c) \\
& \quad=\left[\lambda_{m \nu}(c)+c^{2}\left(1-I_{l_{0}, l_{0}}\right)-l_{0}\left(l_{0}+1\right)\right] / c^{2} I_{l_{0}, l_{0}+2} .
\end{aligned}
$$

Thus, given some value for $\lambda_{m \nu}(c)$, the coefficient ratios in Eqs. (27) and (28) may be obtained by iteration of the continued fraction on the righthand side.

Consideration of the limiting value of the eigenvalue $\lambda_{m \nu}(c)$ for small $c$, given by Eq. (9b), suggests that an appropriate expression to determine $\lambda_{m \nu}(c)$ is Eq. (25) with $l$ set equal to $\nu$ :

$$
\begin{aligned}
\lambda_{m \nu}(c)= & \nu(\nu+1) \\
& +c^{2}\left[I_{\nu, \nu}-1+I_{\nu, \nu-2} D_{\nu-2}^{m \nu}(c) / D_{\nu}^{m \nu}(c)\right. \\
& \left.+I_{\nu, \nu+2} D_{\nu+2}^{m \nu}(c) / D_{\nu}^{m \nu}(c)\right] .
\end{aligned}
$$

B. Calculational procedures

One must first choose an approximate initial value for $\lambda_{m \nu}(c)$. Flammer gives both a pertur- 
bation expansion ${ }^{46}$ for $\lambda_{m \nu}(c)$, valid for $c \leqslant 2$, and an asymptotic expansion ${ }^{47}$ for $\lambda_{m \nu}(c)$, valid for $c$ $z 10$. For intermediate values of $c$ we determined which expansion converged best and used that expansion's estimate as our initial value for $\lambda_{m \nu}(c)$. The initial value for $\lambda_{m \nu}(c)$ is used to determine the two coefficient ratios on the right in Eq. (31) according to the continued-f raction procedure outlined for Eqs. (27) and (28). Substitution of the calculated coefficient ratios on the right in Eq. (31) determines a new value for $\lambda_{m \nu}(c)$, which is then used to redetermine the coefficient ratios. This procedure is carried out iteratively until self-consistency of Eq. (31) is obtained.

Once the exact value for $\lambda_{m \nu}(c)$ is obtained, one may obtain the ratio of the first two coefficients according to Eq. (30). The rest of the coefficient ratios are determined from Eq. (28). The normalization equation (24) determines the coefficients absolutely. Finally, the diagonal second derivative coupling matrix elements are calculated according to [cf. Eqs. (18), (19), and (22)]

$$
\begin{aligned}
\left(g_{m \nu}, \frac{\partial^{2} g_{m \nu}}{\partial r^{2}}\right) & =-\int_{0}^{*}\left(\frac{\partial g_{m \nu}}{\partial r}\right)^{2} \sin \theta d \theta \\
& =-\sum_{l \geqslant|m|}\left(\frac{\partial D_{l}^{m \nu}(c)}{\partial r}\right)^{2} .
\end{aligned}
$$

\section{RESULTS AND DISCUSSION}

We have solved Eq. (20) to obtain rigorous lower bounds on the energies for the hydrogenic levels 1s $m_{l}=0,2 p m_{l}=0$, and $2 p m_{l}= \pm 1$ for magnetic fields in the range $10^{7} \leqslant B \lesssim 10^{11} \mathrm{G}$. We have also solved Eq. (20) to obtain energies for the hydrogenic $2 s m_{l}=0$ level for fields in the range $10^{7} \leqslant B$ $\lesssim 10^{11} \mathrm{G}$. These latter energies are not rigorous lower bounds, of course, since the $2 s$ level is not the lowest level having $m_{l}=0$ and even parity. For the hydrogenic $1 s m_{l}=0$ level we have solved Eq. (21) to obtain rigorous upper bounds on the energy for fields in the range $10^{7} \leqslant B \leqslant 10^{9} \mathrm{G}$. Finally, we have used our calculated energies to present results for the binding energy of each hydrogenic level. Of course, our rigorous lower (upper) bounds on the level energies imply rigorous upper (lower) bounds on the binding energies. For all of these states, we compared our calculated binding energies with the best variational calculations and with calculations by other methods.

\section{A. Numerical details}

We have calculated the oblate-sphe roidal eigenvalues according to the procedure outlined in Sec. IV. Our calculated eigenvalues agree to 8 digits with the 18-digit eigenvalues tabulated by the Naval
Research Laboratory. ${ }^{48}$ Since Eqs. (20) and (21) do not contain first derivatives, we used the Numerov method ${ }^{49}$ to solve these differential equations for the eigenvalues $E^{\prime}$. For zero magnetic field our computed energy eigenvalues agree with the analytically known hydrogen energies to six digits. Our computed binding energies are presented in the tables below to no more than four digits, all of which are expected to be significant in the light of the numerical checks mentioned above. [More specifically, all our level energies are assumed to be accurate to at least four digits; however, the binding energies obtained from Eq. (35) are given in the tables to fewer than four digits, for high magnetic fields, owing to the subtraction of comparably sized numbers on the right in $\mathrm{Eq} .(35)$.

\section{B. Binding energies}

The binding energy of an electron in a Coulomb potential is altered in the presence of a uniform magnetic field. In our treatment the binding energy is obtained by using the asymptotic expan$\operatorname{sion}^{47}$ for the oblate-spheroidal eigenvalue $\lambda_{m \nu}(c)$ :

$\lambda_{m \nu}(c) \underset{c \rightarrow \infty}{\rightarrow}-c^{2}+2 c(2 \mu+m+1)+$ terms of order $c^{0}$,

where

$$
\mu=\left\{\begin{array}{l}
\frac{1}{2}(\nu-m) \text { for } \nu-m \text { even } \\
\frac{1}{2}(\nu-m-1) \text { for } \nu-m \text { odd } .
\end{array}\right.
$$

Substituting Eqs. (33) and (7) into Eq. (20) and letting $r \rightarrow \infty$ (recalling once again that $c \equiv \alpha r^{2}$ ), we obtain the asymptotic limit of Eq. (20):

$$
\left(-\frac{1}{2} \frac{d^{2}}{d \gamma^{2}}+\alpha(2 m+2 \mu+1)\right) h_{m \nu}(r)=E h_{m \nu}(r) \text {. }
$$

The binding energy of the $n$th eigenstate is thus given by

$$
I_{n}=\alpha(2 m+2 \mu+1)-E_{n} .
$$

For the $s$ and $p$ levels considered in this paper, the values of the threshold shift $\alpha(2 m+2 \mu+1)$ are presented in Table II. Subtracting our calculated lower (upper) bounds for the energies $E_{n}$ from the threshold shift, we obtain rigorous upper (lower) bounds on the binding energies $I_{n}$. Note that the

TABLE II. Threshold energy shifts.

\begin{tabular}{ccrrc}
\hline \hline Hydrogenic level & $\nu$ & $m$ & $\mu$ & $\alpha(2 m+2 \mu+1)$ \\
\hline$n s m_{l}=0$ & 0 & 0 & 0 & $\alpha$ \\
$n p m_{l}=0$ & 1 & 0 & 0 & $\alpha$ \\
$n p m_{l}=+1$ & 1 & +1 & 0 & $3 \alpha$ \\
$n p m_{l}=-1$ & 1 & -1 & +1 & $\alpha$ \\
\hline \hline
\end{tabular}




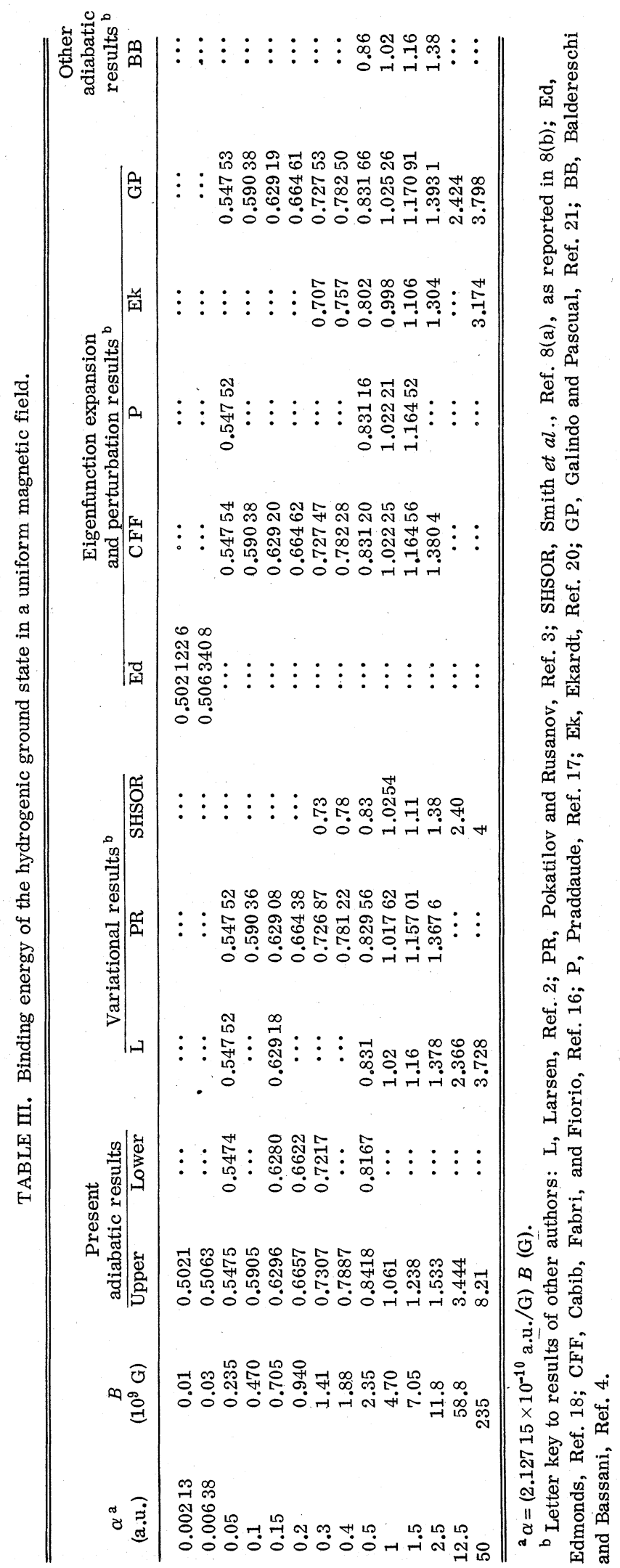


TABLE IV. Binding energy of the hydrogenic $2 p m_{l}=0$ state in a uniform magnetic field.

\begin{tabular}{|c|c|c|c|c|c|c|}
\hline \multirow{2}{*}{$\begin{array}{c}\alpha^{a} \\
\text { (a.u.) }\end{array}$} & \multirow{2}{*}{$\begin{array}{c}B \\
\left(10^{9} \mathrm{G}\right)\end{array}$} & \multirow{2}{*}{$\begin{array}{c}\text { Present } \\
\text { adiabatic results } \\
\text { (upper bound) }\end{array}$} & \multicolumn{2}{|c|}{$\begin{array}{l}\text { Variational } \\
\text { results }\end{array}$} & \multicolumn{2}{|c|}{$\begin{array}{l}\text { Eigenfunction expansion } \\
\text { and perturbation results }\end{array}$} \\
\hline & & & $\mathrm{I}$ & $\mathrm{dSB}$ & $\mathrm{P}$ & GP \\
\hline 0.00213 & 0.01 & 0.1271 & $\cdots$ & $\cdots$ & $\cdots$ & $\cdots$ \\
\hline 0.00638 & 0.03 & 0.1312 & $\cdots$ & $\cdots$ & $\cdots$ & $\cdots$ \\
\hline 0.05 & 0.235 & 0.1629 & 0.16238 & 0.1549 & 0.16241 & 0.16223 \\
\hline 0.1 & 0.470 & 0.1874 & $\cdots$ & $\cdots$ & $\cdots$ & 0.182586 \\
\hline 0.15 & 0.705 & 0.2061 & $\cdots$ & $\cdots$ & $\cdots$ & 0.194066 \\
\hline 0.2 & 0.940 & 0.2215 & $\cdots$ & $\cdots$ & $\cdots$ & 0.201042 \\
\hline 0.3 & 1.41 & 0.2465 & $\cdots$ & $\cdots$ & $\cdots$ & 0.208833 \\
\hline 0.4 & 1.88 & 0.2669 & $\cdots$ & $\cdots$ & $\cdots$ & 0.21299 \\
\hline 0.5 & 2.35 & 0.2843 & 0.2596 & 0.2594 & 0.26000 & 0.21556 \\
\hline 1 & 4.70 & 0.3485 & $\cdots$ & 0.296 & 0.29770 & 0.22081 \\
\hline 1.5 & 7.05 & 0.395 & $\cdots$ & 0.308 & 0.3200 & 0.2226 \\
\hline 2.5 & 11.8 & 0.463 & $\cdots$ & $\cdots$ & $\cdots$ & 0.224 \\
\hline 12.5 & 58.8 & 0.80 & $\cdots$ & $\cdots$ & $\cdots$ & 0.226 \\
\hline 50 & 235 & 1.37 & $\cdots$ & $\cdots$ & $\cdots$ & 0.226 \\
\hline
\end{tabular}

${ }^{a} \alpha=\left(2.12715 \times 10^{-10}\right.$ a.u./G) $B(G)$.

${ }^{b}$ Letter key to results of other authors: L, Larsen, Ref. 2; dSB, dos Santos and Brandi, Ref. 12; P, Praddaude, Ref. 17; GP, Galindo and Pascual, Ref. 21.

binding energies of the $2 p m_{l}= \pm 1$ levels are degenerate, since the difference in the threshold shift of $2 \alpha$ exactly cancels the difference of the same amount in the energy levels..$^{50}$

Our lower and upper bounds for the hydrogenic $1 s$ binding energy are presented in Table III. Our lower-bound results agree to at least two digits with the results of the best variational calculations $\mathrm{s}^{2,3,8}$ up to fields of $10^{9} \mathrm{G}$. For fields of order $10^{8} \mathrm{G}$ or lower, our lower-bound results are essentially identical to our upper-bound results . For fields of order $10^{7} \mathrm{G}$, our upper-bound results agree very closely with the highly accurate Sturmian-function expansion results of Edmonds..$^{18}$ For fields up to $10^{9} \mathrm{G}$ our upper-bound results agree to at least two digits with the results of other calculations.

Comparison of our adiabatic upper-bound results (based on spherical coordinates) with the adiabatic upper-bound results of Balderschi and Bassani ${ }^{4}$ (based on cylindrical coordinates) proves instructive. As shown in Table III, for $\alpha=0.5(B=2.35$ $\times 10^{9} \mathrm{G}$ ) our upper bound is lower than that of Ref. 4. For $\alpha=1\left(B=4.70 \times 10^{9} \mathrm{G}\right)$, however, the situation is reversed and the upper bound of Ref. 4 is lower than our upper bound. These facts suggest that $\alpha \simeq 1$ is the dividing line-at least as far as the 1 s energy level is concerned-between lower fields (i.e., $B \lesssim 10^{9} \mathrm{G}$ ) for which a spheroidal-coordinate description is suitable and higher fields

TABLE V. Binding energy of the hydrogenic $2 p m_{l}= \pm 1$ state in a uniform magnetic field.

\begin{tabular}{|c|c|c|c|c|c|c|c|}
\hline \multirow{2}{*}{$\begin{array}{c}\alpha^{\mathrm{a}} \\
(\mathrm{a} . \mathrm{u} .)\end{array}$} & \multirow{2}{*}{$\begin{array}{c}B \\
\left(10^{9} \mathrm{G}\right)\end{array}$} & \multirow{2}{*}{$\begin{array}{c}\text { Present } \\
\text { adiabatic results } \\
\text { (upper bound) }\end{array}$} & \multicolumn{3}{|c|}{ Variational results ${ }^{b}$} & \multicolumn{2}{|c|}{$\begin{array}{l}\text { Eigenfunction expansion } \\
\text { and perturbation results }\end{array}$} \\
\hline & & & $\mathrm{L}$ & $\mathrm{dSB}$ & $\mathrm{BNW}$ & $\mathrm{P}$ & GP \\
\hline 0.00213 & 0.01 & 0.1292 & $\cdots$ & $\cdots$ & $\cdots$ & $\cdots$ & $\cdots$ \\
\hline 0.00638 & 0.03 & 0.1372 & $\cdots$ & $\cdots$ & $\cdots$ & $\cdots$ & $\cdots$ \\
\hline 0.05 & 0.235 & 0.2013 & 0.20081 & 0.1862 & $\cdots$ & 0.20084 & 0.200194 \\
\hline 0.1 & 0.470 & 0.2530 & $\cdots$ & $\cdots$ & $\cdots$ & $\cdots$ & 0.242423 \\
\hline 0.15 & 0.705 & 0.2947 & $\cdots$ & $\cdots$ & $\cdots$ & $\cdots$ & 0.26652 \\
\hline 0.2 & 0.940 & 0.3308 & $\cdots$ & $\cdots$ & $\cdots$ & $\cdots$ & 0.28126 \\
\hline 0.3 & 1.41 & 0.3929 & $\cdots$ & $\cdots$ & $\cdots$ & $\cdots$ & 0.29780 \\
\hline 0.4 & 1.88 & 0.4468 & $\cdots$ & $\cdots$ & $\cdots$ & $\cdots$ & 0.30665 \\
\hline 0.5 & 2.35 & 0.4954 & 0.456 & 0.4540 & 0.45396 & 0.45659 & 0.3121 \\
\hline 1 & 4.70 & 0.6967 & $\cdots$ & 0.5977 & 0.5947 & 0.59958 & 0.3233 \\
\hline 1.5 & 7.05 & 0.8629 & $\cdots$ & 0.702 & $\cdots$ & 0.70352 & 0.3271 \\
\hline 2.5 & 11.8 & 1.148 & 0.86 & 0.92 & $\cdots$ & . & 0.3301 \\
\hline 12.5 & 58.8 & 3.20 & 1.60 & 1.51 & $\cdots$ & $\cdots$ & 0.334 \\
\hline 50 & 235 & 9.1 & 2.60 & -0.1 & 2.56 & $\cdots$ & 0.33 \\
\hline
\end{tabular}

${ }^{\mathrm{a}} \alpha=\left(2.12715 \times 10^{-10}\right.$ a.u./G) $B(\mathrm{G})$.

${ }^{b}$ Letter key to results of other authors: L, Larsen, Ref. 2; dSB, dos Santos and Brandi, Ref. 12; BNW, Bhaduri, Nogami, and Warke, Ref. 13; P, Praddaude, Ref. 17; GP, Galindo and Pascual, Ref. 21. 


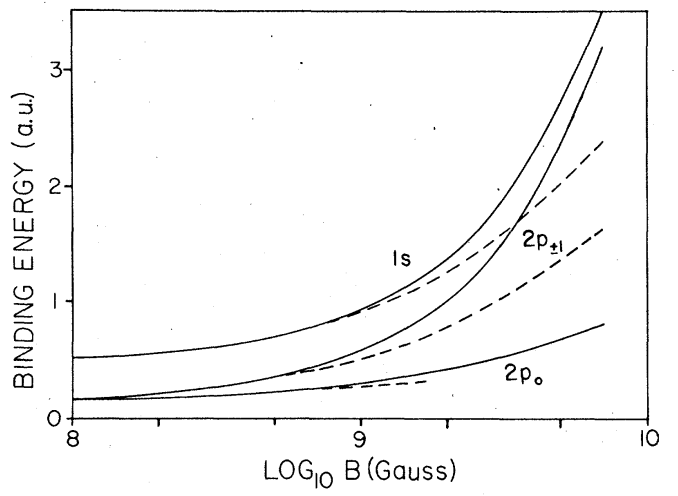

FIG. 1. Upper and lower bounds for the hydrogenic $1 s$ and $2 p$ binding energies in a uniform magnetic field. Solid lines, present adiabatic results giving upper bounds on the binding energies; dashed lines, best variational results giving lower bounds on the binding energies (cf. Tables III-V).

(i.e., $B \gtrsim 10^{9} \mathrm{G}$ ) for which a cylindrical-coordinate description is suitable.

Our adiabatic upper-bound results for the hydrogenic $2 p$ binding energies for $m_{l}=0$ and \pm 1 are presented in Tables IV and $V$. The number of other calculations for these binding energies is considerably fewer than for the 1 s level. For $\alpha$ $=0.05\left(B=2.35 \times 10^{8} \mathrm{G}\right)$ our results agree with those of other calculations. For $\alpha=0.5(B=2.35$ $\times 10^{9} \mathrm{G}$ ) our upper-bound results are significantly higher than the variational lower-bound results. For values of $\alpha$ between 0.05 and 0.5 , our results do not agree very closely with the results of Ref. 21.

Figure 1 presents a summary of the binding-energy calculations discussed thus far. The solid lines give our upper-bound results for the $1 s$ and $2 p$ binding energies. The dashed lines present the highest variational lower-bound result at each field strength, as given in Tables III-V. Clearly the standard for any future calculations is that the results fall between the limits shown graphically in Fig. 1 and presented numerically in the tables.

It is of interest to compare our adiabatic results [obtained using energies calculated from Eq. (20)] for the hydrogenic $2 s$ binding energy with calculations of other authors. We emphasize that neither our results nor the adiabatic results of Ref. 4 are rigorous upper bounds for the $2 s$ binding energy. However, Table VI shows that for magnetic fields of order $10^{7} \mathrm{G}$, our results agree very closely with the very accurate results of Edmonds..$^{18}$ Even for fields of order $10^{10} \mathrm{G}$ our results agree to two digits with those of some of the other calculations.
C. Level energies

In our calculations we obtain the reduced energies $E^{\prime}$ directly. These are related to the level energies by Eq. (7). We have chosen to present our results, however, in the form of binding energies, which are more sensitive tests of alternative calculational procedures for high magnetic fields. The reader may, for course, obtain our results for the level energies through the use of Eq. (35), the value of the threshold shift in Table II, and our values of $I_{n}$ presented in Tables III-VI. Figure 2 summarizes most of our energy-level results. Our rigorous lower bounds on the hydrogen $1 s$ and $2 p$ energy levels are shown as the solid lines in Fig. 2. The best variational upper bounds on the energy levels from Tables III-V are shown as the dashed lines in Fig. 2. As with the binding energies, the standard for future energy-level calculations for the $1 s$ and $2 p$ energy levels of hydrogen is that they lie between the lower and upper bounds shown in Fig. 2 .

\section{Conclusions}

We have presented results of adiabatic calculations in spherical coordinates which provide upper bounds on the binding energies of the lowest hydrogenic energy levels in a uniform magnetic field. Results of an adiabatic lower-bound calculation for the ground-state binding energy have also been presented. Our results agree with other variational, adiabatic, and eigenfunction-expansion calculations for magnetic fields up to $10^{9} \mathrm{G}$. For higher fields, our results differ from those of other calculations, thus indicating that the coupling matrix elements between different adiabatic states can no longer be neglected. Though this paper has concentrated on field strengths in the range $10^{7} \leqslant B \leqslant 10^{11}$, the close agreement between our calculated energy levels and the very accurate results of Edmonds ${ }^{18}$ for $B \simeq 10^{7} \mathrm{G}$ indicates that for fields $B \lesssim 10^{7} \mathrm{G}$, one may obtain reliable energy levels simply by solving the differential equation (20). We expect also that the use of a sphericalcoordinate approach provides accurate wave functions in the vicinity of the origin, where optical absorption takes place.

\section{ACKNOWLEDGMENTS}

This work has benefited greatly from the numerous discussions, advice, and encouragement given by Professor Ugo Fano and Professor Joseph Macek. In particular, we wish to thank Professor Fano for suggesting the spherical-coordinate approach adopted in this paper, and Professor Macek for pointing out to us the oblate- 


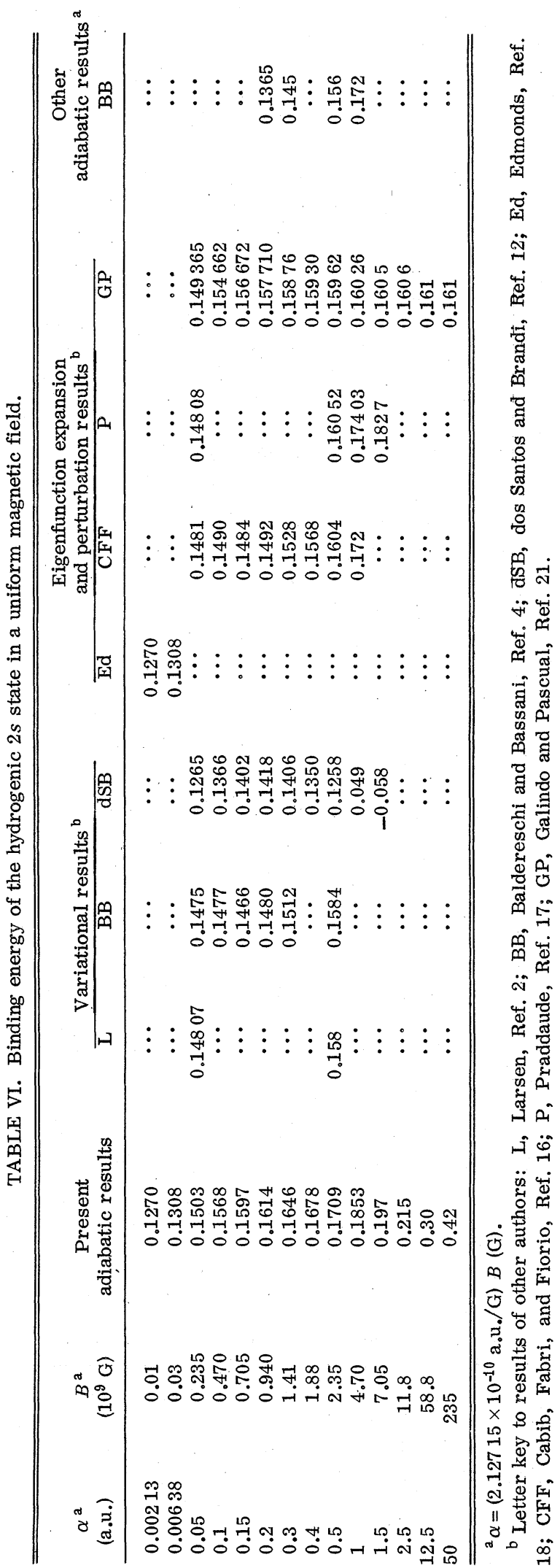

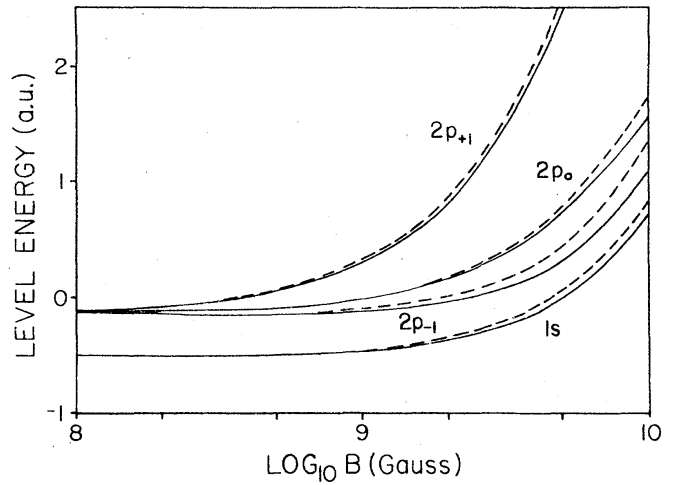

FIG. 2. Upper and lower bounds for the hydrogenic $1 s$ and $2 p$ level energies in a uniform magnetic field. Solid lines, present lower-bound adiabatic results; dashed lines, best variational upper-bound results (cf. Tables III-V).

spheroidal angle functions. We are grateful also to Dr. S. Hanish for providing us with six volumes of tabulated values for the spheroidal functions and eigenvalues, which proved invaluable in checking our computer programs. Lastly, we wish to thank Dr. C. Flammer for informing us of the work of the Naval Research Laboratory concerning spheroidal functions. This work was supported by the U.S. Department of Energy under Contract No. EY-76-S-02-2892.A002. One of us (AFS) would like to thank the Alfred P. Sloan Foundation for its support.

\section{APPENDIX}

We wish to prove that the lower- (upper-) bound adiabatic approximation presented in Sec. III is indeed a lower (upper) bound on the lowest exact energy for each symmetry state of the system. These theorems are analogous to ones in molecular physics which state that the Born-Oppenheimer approximation gives a lower bound on molecular energy levels, and that the adiabatic approximation (in which diagonal coupling terms are included) gives an upper bound on the molecular energy levels. ${ }^{51,52}$ Our proof therefore follows the proof given for the molecular problem by Brattsev..$^{51}$

Consider the expectation value of the Hamiltonian for an electron in combined Coulomb and uniform magnetic fields [cf. Eq. (5)]:

$$
\begin{aligned}
E_{\text {exact }}^{\prime}=\int_{0}^{\infty} d r \int_{0}^{\pi} & \sin \theta d \theta \chi_{m}^{*}(r, \theta) \\
& \times\left(-\frac{1}{2} \frac{d^{2}}{d r^{2}}-\frac{1}{r}+\frac{\Lambda_{m}\left(\alpha r^{2}, \theta\right)}{\partial r^{2}}\right) \chi_{m}(r, \theta),
\end{aligned}
$$

where $E_{\text {exact }}^{\prime}$ is the exact energy of the system in 
the state characterized by the exact wave function $\chi_{m}(r, \theta)$. The symmetry of the state is determined by the $z$ component of the orbital angular momentum, denoted by $m$, and by the parity, which is not explicitly indicated. One may always rewrite the wave function as

$$
\chi_{m}(r, \theta) \equiv F_{m}(r) G_{m}(r, \theta),
$$

where the normalization is chosen such that

$$
\begin{aligned}
& \int_{0}^{\pi}\left|G_{m}(r, \theta)\right|^{2} \sin \theta d \theta=1, \\
& \int_{0}^{\infty}\left|F_{m}(r)\right|^{2} d r=1 .
\end{aligned}
$$

Substitution of Eq. (A2) into Eq. (A1) gives

$$
\begin{aligned}
E_{\mathrm{exact}}^{\prime}=\int_{0}^{\infty} & d r F_{m}^{*}(r) \\
& \times\left(-\frac{1}{2} \frac{d^{2}}{d r^{2}}-\frac{1}{r}+U_{m}(r)+V_{m}(r)\right) F_{m}(r),
\end{aligned}
$$

where we have defined

$U_{m}(r) \equiv \frac{1}{2 r^{2}} \int_{0}^{\pi} \sin \theta d \theta G_{m}^{*}(r, \theta) \Lambda_{m}\left(\alpha r^{2}, \theta\right) G_{m}(r, \theta)$,

$V_{m}(r) \equiv-\frac{1}{2} \int_{0}^{\pi} \sin \theta d \theta G_{m}^{*}(r, \theta) \frac{\partial^{2} G_{m}(r, \theta)}{\partial r^{2}}$.

Note that in deriving Eq. (A5) we have used the result

$$
\int_{0}^{\pi} \sin \theta d \theta G_{m}^{*}(r, \theta) \frac{\partial G_{m}(r, \theta)}{\partial r}=0
$$

which follows directly upon differentiation of the normalization equation (A3) with respect to $r$.

\section{A. Proof of upper-bound theorem}

Equation (A5) gives the exact system energy $E_{\text {exact }}^{\prime}$ in terms of the expectation value of the Hamiltonian obtained with the exact wave function $\chi_{m}(r, \theta)$, which we have chosen to write as in Eq. (A2). If, however, we had chosen an approximate $\chi_{m}(r, \theta)$, then according to the Rayleigh-Ritz principle the right-hand side of Eq. (A5) would be an upper bound on the lowest energy of the system for each symmetry state. In particular, if we had chosen $G_{m}(r, \theta)$ to be the oblate-spheroidal angle function $g_{m \nu}(c, \theta)$, where $\nu$ denotes the smallest eigenvalue of $\Lambda_{m}(c, \theta)$ and $c \equiv \alpha r^{2}$, then Eq. (A6) becomes [cf. Eqs. (8) and (A3)]

$$
U_{m}(r)=\left[\lambda_{m \nu}(c)+c^{2}\right] / 2 r^{2}
$$

When Eq. (A9) is then substituted into Eq. (A5), we obtain

$$
\begin{aligned}
E_{\text {exact }}^{\prime \leqslant \int_{0}} d r F_{m \nu}^{*}(r)[ & -\frac{1}{2} \frac{d^{2}}{d r^{2}}-\frac{1}{r}+\frac{\lambda_{m \nu}(c)+c^{2}}{2 r^{2}} \\
& \left.-\frac{1}{2}\left(g_{m \nu}, \frac{\partial^{2} g_{m \nu}}{\partial r^{2}}\right)\right] F_{m \nu}(r) .
\end{aligned}
$$

If $F_{m \nu}(r)$ is now chosen to be the eigenfunction corresponding to the lowest eigenvalue of the operator in brackets in Eq. (A10), then we obtain the upper-bound adiabatic approximation of Sec. III [cf. Eq. (21)].

\section{B. Proof of lower-bound theorem}

Returning to the exact result in Eq. (A5), we note that the potential $V_{m}(r)$ [cf. Eq. (A7)] is positive definite, since

$$
\begin{aligned}
-\int_{0}^{\pi} \sin \theta d \theta G_{m}^{*} & (r, \theta) \frac{\partial^{2} G_{m}(r, \theta)}{\partial r^{2}} \\
& =+\int_{0}^{\pi} \sin \theta d \theta\left|\frac{\partial G_{m}(r, \theta)}{\partial r}\right|^{2} \geqslant 0 .
\end{aligned}
$$

Equation (A11) follows directly upon differentiation of Eq. (A8) with respect to $r$. Thus if we drop $V_{m}(r)$ from Eq. (A5), we obtain

$$
E_{\text {exact }}^{\prime} \geqslant \int_{0}^{\infty} d r F_{m}^{*}(r)\left(-\frac{1}{2} \frac{d^{2}}{d r^{2}}-\frac{1}{r}+U_{m}(r)\right) F_{m}(r) .
$$

Consider now the potential $U_{m}(r)$ defined by the expectation value in Eq. (A6). This expectation value is always greater than the smallest eigenvalue of $\Lambda_{m}\left(\alpha r^{2}, \theta\right)$, denoted by the index $\nu$ :

$$
U_{m}(r) \geqslant\left[\lambda_{m \nu}(c)+c^{2}\right] / 2 r^{2}
$$

Substituting this inequality into Eq. (A12) gives

$$
\begin{aligned}
E_{\text {exact }}^{\prime} \geqslant & \int_{0}^{\infty} d r F_{m}^{*}(r)\left(-\frac{1}{2} \frac{d^{2}}{d r^{2}}-\frac{1}{r}+\frac{\lambda_{m \nu}(c)+c^{2}}{2 r^{2}}\right) \\
& \times F_{m}(r) .
\end{aligned}
$$

Finally, the expectation value on the right in Eq. (A14) is always greater than the least eigenvalue of the operator in parentheses. But the least eigenvalue of this operator is obtained by solving Eq. (20); i.e., it is obtained by the lower-bound adiabatic approximation of Sec. III. 
${ }^{1}$ Y. Yafet, R. W. Keyes, and E. N. Adams, J. Phys. Chem. Solids 1, 137 (1956).

${ }^{2}$ D. M. Larsen, J. Phys. Chem. Solids 29, 271 (1968).

${ }^{3}$ E. P. Polatilov and M. M. Rusanov, Fiz. Tverd. Tela (Leningrad) 10, 3117 (1968) [Sov. Phys. Solid State $10,2458(19 \overline{69)}]$.

${ }^{4} \mathrm{~A}$. Baldereschi and F. Bassani, in Proceedings of the 10 th International Conference on the Physics of Semiconductors, Cambridge, Mass., AEC Oak Ridge CONF-700801, edited by S. P. Keller, J. C. Hensel, and F. Stern (U. S. AEC., Washington, D.C., 1970), pp. 191-196.

${ }^{5} \mathrm{R}$. Cohen, J. Lodenquai, and M. Ruderman, Phys. Rev. Lett. 25, 467 (1970).

${ }^{6} \mathrm{~J}$. Callaway, Phys. Lett. A 40, 331 (1972).

${ }^{7}$ A. K. Rajagopal, G. Chanmugam, R. F. O'Connell, and G. L. Surmelian, Astrophys. J. 177, 713 (1972).

${ }^{8}$ (a) E. R. Smith, R. J. W. Henry, G. L. Surmelian, R. F. O'Connell, and A. K. Rajogopal, Phys. Rev. D 6, 3700 (1972); (b) G. L. Surmelian and R. F. O'Connell, Astrophys. J. 190, 741 (1974).

${ }^{9} \mathrm{~L}$. W. Wilson, Astrophys. J. 188, 349 (1974).

${ }^{10}$ H. S. Brandi, Phys. Rev. A $\frac{11}{11} 1835$ (1975).

${ }^{11}$ A. R. P. Rau and L. Spruch, Astrophys. J. 207, 671 (1976).

${ }^{12}$ R. R. dos Santos and H. S. Brandi, Phys. Rev. A $\underline{13}$, 1970 (1976).

${ }^{13}$ R. K. Bhaduri, Y. Nogami, and C. S. Warke, Astrophys. J. 217, 324 (1977).

${ }^{14} \mathrm{~J}$. M. Wadehra, Astrophys. J. 226, 372 (1978).

${ }^{15}$ L. I. Schiff and H. Snyder, Phys. Rev. 55, 59 (1939).

${ }^{16}$ D. Cabib, E. Fabri, and G. Fiorio, Solid State Commun. 9,1517 (1971).

${ }^{17}$ H. C. Praddaude, Phys. Rev. A 6, 1321 (1972).

${ }^{18}$ A. R. Edmonds, J. Phys. B $\underline{6}, 1 \overline{6} 03$ (1973).

${ }^{19}$ R. H. Garstang and S. B. Kemic, Astrophys. Space Sci. 31, 103 (1974).

${ }^{20}$ W. Ekardt, Solid State Commun. 16, 233 (1975).

${ }^{21} \mathrm{~A}$. Galindo and P. Pascual, Nuovo Cimento B 34, 155 (1976).

${ }^{22}$ R. J. Elliott and R. Loudon, J. Phys. Chem. Solids 15, 196 (1960).

${ }^{23}$ H. Hasegawa and R. E. Howard, J. Phys. Chem. Solids 21,179 (1961).

${ }^{24} \mathrm{~A}$. G. Zhilich and B. S. Monozon, Fiz. Tverd. Tela (Leningrad) 8 , 3559 (1966) [Sov. Phys. Solid State $\underline{8}$, 2846 (1967)].

${ }^{25}$ V. Canuto and D. C. Kelly, Astrophys. Space Sci. 17, 277 (1972).

${ }^{26}$ Rigorous upper bounds on excited energy levels may also be obtained by variational methods, but only at the cost of much greater computational labor. See, e.g., the work of E. A. Hylleraas and B. Undheim, Z. Phys. 65, 759 (1930), as well as the discussion of E. C. Kemble, The Fundamental Principles of Quantum Mechanics (McGraw-Hill, New York, 1937), Sec. 51C. ${ }^{27}$ U. Fano, Colloq. Int. CNRS 273, 127 (1977).

${ }^{28}$ A. F. Starace and G. L. Webster, Bull. Am. Phys. Soc. 22,1335 (1977).

${ }^{29}$ C. Flammer, Spheroidal Wave Functions (Stanford University, Stanford, Calif., 1957).

${ }^{30} \mathrm{M}$. Abramowitz and I. A. Stegun, Handbook of Mathe- matical Functions (Dover, New York, 1965).

${ }^{31}$ W. R. S. Garton and F. S. Tomkins, Astrophys. J. 158, 839 (1969).

${ }^{32}$ R. J. Fonck, F. L. Roesler, D. H. Tracy, K. T. Lu, F. S. Tomkins, and W. R. S. Garton, Phys. Rev. Lett. 39,1513 (1977); 40, 201 (1978).

${ }^{33}$ M. L. Zimmerman, J. C. Castro-Neto, and D. Kleppner, Phys. Rev. Lett. 40, 1083 (1978).

${ }^{34}$ R. J. Fonck, D. H. Tracy, D. C. Wright, and F. S. Tomkins, Phys. Rev. Lett. 40, 1367 (1978).

${ }^{35}$ K. T. Lu, F. S. Tomkins, and W. R. S. Garton, Proc. Roy. Soc. London Ser. A 362, 421 (1978).

${ }^{36}$ G. L. Webster and A. F. Starace, Bull. Am. Phys. Soc. 23,1103 (1978).

${ }^{37}$ U. Fano and A. F. Starace (unpublished).

${ }^{38}$ A. R. Edmonds, J. Phys. (Paris) Colloq. C4 31, 71 (1970).

${ }^{39}$ A. F. Starace, J. Phys. B 6, 585 (1973).

${ }^{40}$ R. H. Garstang, Rep. Prog. Phys. 40, 105 (1977).

${ }^{41} \mathrm{H}$. Hasegawa, in Physics of Solids in Intense Magnetic Fields, edited by E. D. Haidemenakis (Plenum, New York, 1969), Chap. 10.

${ }^{42}$ R. Gajewski, Physica (Amsterdam) 47, 575 (1970).

${ }^{43} \mathrm{H}$. A. Bethe and E. E. Salpeter, Quantum Mechanics of One-and Two-Electron Atoms (Springer, Berlin, 1957), Secs. 45-47.

${ }^{44}$ In this paper we assume the atomic nucleus to be infinitely massive. W. E. Lamb [Phys. Rev. 85, 259 (1952)] has investigated the influence of nuclear motion on the linear Zeeman energy shift (see also Ref. 43, p. 214). Furthermore, M. Ryvkin has shown for a hydrogenlike atom in a uniform magnetic field that the center-of-mass energy is not conserved separately from the energy of relative motion: Dokl. Akad. Nauk SSSR 221, 67 (1975) [Sov. Phys. Dokl. 20, 192 (1975)]. Very recently, J. T. Virtamo and J. T. A. Simola [Phys. Lett. A 66, 371 (1978)] have estimated the influence of nuclear motion on electron binding energies in atomic hydrogen for magnetic fields above $5 \times 10^{10} \mathrm{G}$.

${ }^{45}$ One way to evaluate the integral in $\mathrm{Eq} .(26)$ is to write $\sin ^{2} \theta$ in terms of the spherical harmonics $Y_{00}(\Omega)$ and $Y_{20}(\Omega)$. The integral of a product of three spherical harmonics is given in terms of $3-j$ symbols by M. Rotenberg et al., The 3-j and 6-j Symbols (Technology, Cambridge, Mass., 1959), Eq. 1-19. Analytic expressions for the 3-j symbols are given by A. R. Edmonds, $A n-$ gular Momentum in Quantum Mechanics, 2nd ed. (Princeton University, Princeton, N. J., 1974), pp. 126-127.

${ }^{46}$ Reference 29, Sec. 3.1.2.

${ }^{47}$ Reference 29 , Sec. 8.2.

${ }^{48}$ S. Hanish, R. V. Baier, A. L. Van Buren, and B. J. King, NRL Report No. 7093, 1970 (unpublished).

${ }^{49} \mathrm{~K}$. Smith, The Calculation of Atomic Collision Processes (Wiley, New York, 1971), Sec.1.4.

${ }^{50}$ Put another way, the energies $E^{\prime}$ calculated from Eq. (5) are independent of the sign of $m$, as shown by Eq. (6).

${ }^{51}$ V. K. Brattsev, Dokl. Akad. Nauk SSSR 160, 570 (1965) [Sov. Phys. Dok1. 10, 44 (1965)].

${ }^{52}$ M. G. Sucre, F. Goychman, and R. Lefebvre, Phys. Rev. A 2, 1738 (1970). 